Article

\title{
Thick-Film Carbon Dioxide Sensor via Anodic Adsorbate Stripping Technique and Its Structural Dependence
}

\author{
Kanokorn Photinon ${ }^{1}$, , Shih-Han Wang ${ }^{2}$ and Chung-Chiun Liu ${ }^{1}$ \\ 1 Chemical Engineering Department, Case Western Reserve University, 10900 Euclid Ave., \\ Cleveland OH 44106, USA; E-Mail: cx19@ case.edu (C.C.L.) \\ 2 Chemical Engineering Department, I-Shuo University, 1, Sec. 1, Syuecheng Rd., Dashu Township, \\ Kaohsiung County 840, Taiwan; E-Mail: shwang@ mail.isu.edu.tw (S.H.W.) \\ * Author to whom correspondence should be addressed; E-Mail: kanokorn@ gmail.com; \\ Tel.: +66-86-921-7373; Fax: +66-53-948-238.
}

Received: 30 July 2009; in revised form: 27 August 2009 / Accepted: 31 August 2009 /

Published: 9 September 2009

\begin{abstract}
A three-electrode based $\mathrm{CO}_{2}$ sensor was fabricated using thick-film technology. The performance of this sensor was further enhanced by incorporating platinum nanoparticles onto the working electrode surface. An eight-fold increase in the signal output was obtained from the electrode with the platinum nanoparticles. The sensing output was linearly related to the $\mathrm{CO}_{2}$ presented. Stability measurements demonstrated that the decline of the active surface area and the sensitivity of the sensor were $8 \%$ and $13 \%$, respectively, over a two week period of time. The sensor response appeared to be a structural dependence of the crystallographic orientation of platinum electrode.
\end{abstract}

Keywords: carbon dioxide sensor; anodic adsorbate stripping; thick-film; platinum nanoparticles; structural dependence

\section{Introduction}

Monitoring and control of carbon dioxide $\left(\mathrm{CO}_{2}\right)$ in the atmosphere are needed due to heightened environmental concerns. Consequently, the development of reliable $\mathrm{CO}_{2}$ monitoring techniques is scientifically and economically important. Various $\mathrm{CO}_{2}$ analytical methods are currently utilized in laboratory experiments and field observations. Infrared sensors, conductivity sensors, and Severinghaus-type sensors (sensors based on a glass $\mathrm{pH}$ electrode covered with a glass permeable 
membrane) for the detection of $\mathrm{CO}_{2}$ are commercially available [1]. However, these sensors have limitations due to the interferences and the non-linear response to $\mathrm{CO}_{2}$ content. With well-defined oxidation and reduction potential for the particular species, amperometric sensors can be used to minimize interferences. Amperometric $\mathrm{CO}_{2}$ sensors have been developed for $\mathrm{CO}_{2}$ sensing and studied extensively, but a complication with hydrogen evolution has prevented amperometric $\mathrm{CO}_{2}$ sensors from reaching their commercial potential. The approach of altering the electrochemistry of $\mathrm{CO}_{2}$ by reacting it with selected complex species [2] has also been unsuccessful in practical $\mathrm{CO}_{2}$ sensing. An anodic adsorbate stripping technique of the adsorbed $\mathrm{CO}_{2}$ was developed to provide a linear dependence on $\mathrm{CO}_{2}$ content. The technique was based on forming adsorbed $\mathrm{CO}_{2}$ and then oxidizing the previously formed adsorbate. The $\mathrm{CO}_{2}$ product that adsorbs at low potential will be referred to as Pt-HCOO throughout this manuscript. Although the true structure of the Pt-HCOO is unclear, its linear response is sufficient for quantitative study as a Pt-HCOO sensor in our specific applications.

The adsorption of carbon dioxide on platinum $(\mathrm{Pt})$ electrodes from acid solutions has been known for more than 40 years. In acidic medium, $\mathrm{CO}_{2}$ only adsorbs onto hydrogen previously adsorbed on a platinum surface $(\mathrm{Pt}-\mathrm{H})$. Numerous investigations and studies had been carried out on this phenomenon, but the structure of Pt-HCOO was not consistent and had been the subject of controversy [3-12]. According to the results obtained with various nonelectrochemical techniques (IR, mass spectrometry, radiochemical methods), the following structures of the $\mathrm{Pt}-\mathrm{HCOO}$ radicals were proposed: $-\mathrm{COOH}$ [5-7,13,14], $-\mathrm{COH} \quad[8,9,14], \quad-\mathrm{HCO} \quad[12]$, linearly or bridged bonded - $\mathrm{CO}[11,15-18]$. The investigation of the mechanism and the Pt-HCOO radicals are beyond the scope of this work. Nevertheless, it is feasible to detect the Pt-HCOO on a platinum surface in an acidic medium as a means to quantify $\mathrm{CO}_{2}$ in a gaseous test environment, regardless of any knowledge about the true structures of the Pt-HCOO radicals. The relationship between the oxidation current and the $\mathrm{CO}_{2}$ content is linear, suggesting a very desirable and sensitive $\mathrm{CO}_{2}$ detection.

Various manufacturing processes can be used to fabricate an electrochemical based $\mathrm{CO}_{2}$ sensor. In this study, the $\mathrm{CO}_{2}$ sensor prototype was fabricated using a thick-film screen printing technique. Thick-film screen printing produces geometrically well-defined, highly reproducible sensor structures in a cost-effective manner. The sensor operated in the anodic adsorbate stripping mode, and the platinum nanoparticles were incorporated onto the working electrode enhancing the sensor performance. Our sensor can thus offer a device for $\mathrm{CO}_{2}$ detection with high performance and at a relatively modest cost.

\section{Results and Discussion}

\subsection{Anodic Adsorbate Stripping Response}

The potential stepped program is illustrated in Figure 1. The adsorption of $\mathrm{CO}_{2}$ on Pt started when the potential was stepped down (step B) to the adsorbed hydrogen potential. Once the potential was changed to where oxidation of the remaining $\mathrm{Pt}-\mathrm{H}$ occurs (step $\mathrm{C}$ ), the anodic current increased accordingly. The contribution of the anodic current from the oxidation of $\mathrm{Pt}-\mathrm{HCOO}$ took place when its oxidation potential was reached; in this case, the oxidation potential was $+0.75 \mathrm{~V}$ (step D). The oxidation charged for $\mathrm{Pt}-\mathrm{HCOO}\left(\mathrm{Q}_{\mathrm{HCOO}}\right)$ could then be determined from the area under the curve from 
this step. Finally, large anodic current was produced from the formation of surface oxides when the potential was stepped to $+1.60 \mathrm{~V}$ (step E).

Figure 1. Potential step program and i-t curve response for the anodic stripping technique, (---) i-t curve response, (-) potential step program.

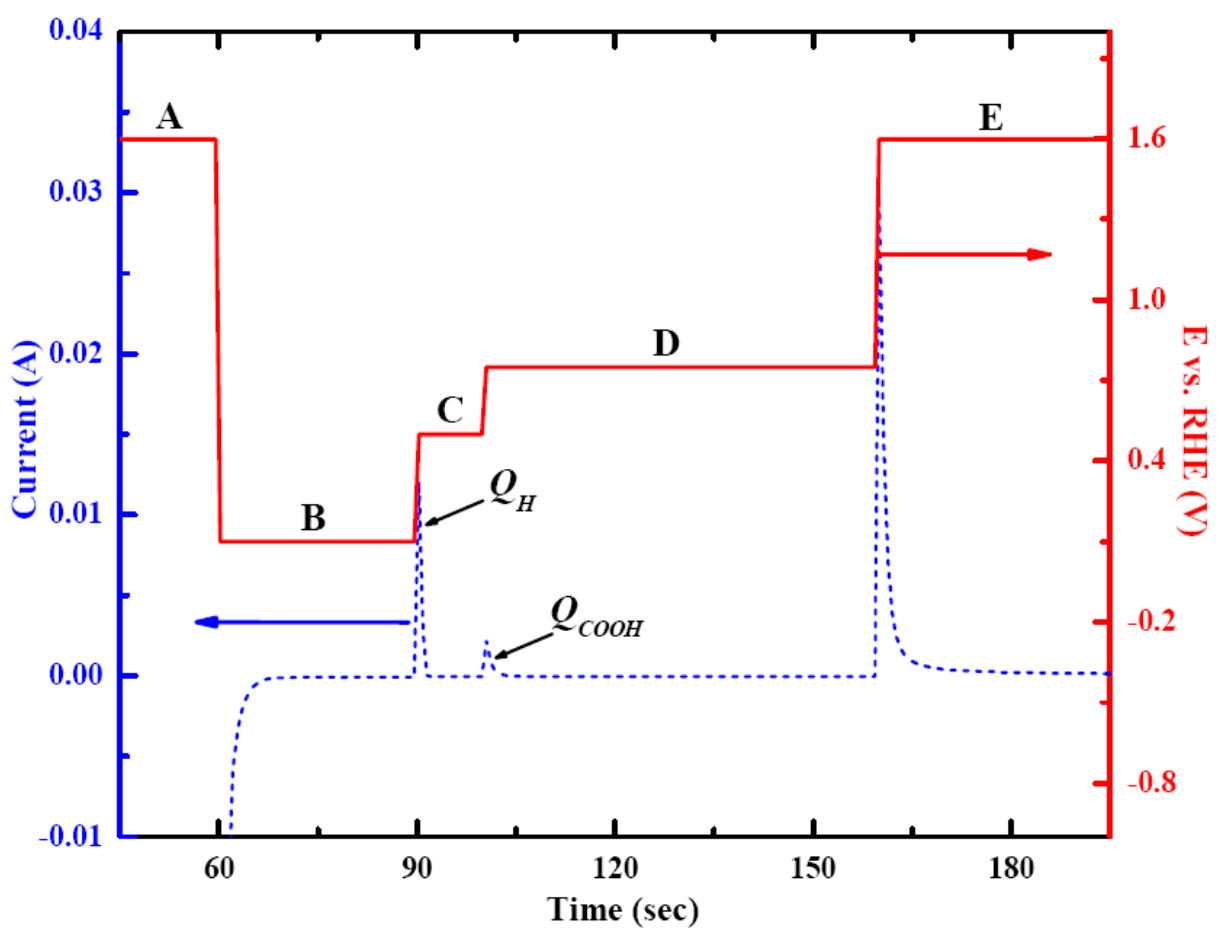

Figure 2. Anodic stripping response for various $\% \mathrm{CO}_{2}$ in gas sample on a $\mathrm{Pt}$-nano electrode demonstrating the inverse relationship between $Q_{H}$ and $Q_{H C O O},(-\square) 0.0 \% \mathrm{CO}_{2}$, $\left.(-\bullet) 0.5 \% \mathrm{CO}_{2},\left(-\Delta_{-}\right) 1.0 \% \mathrm{CO}_{2},(-\boldsymbol{\nabla}) 2.0 \% \mathrm{CO}_{2},(-)\right) 3.0 \% \mathrm{CO}_{2},(-4-) 5.0 \% \mathrm{CO}_{2}$.

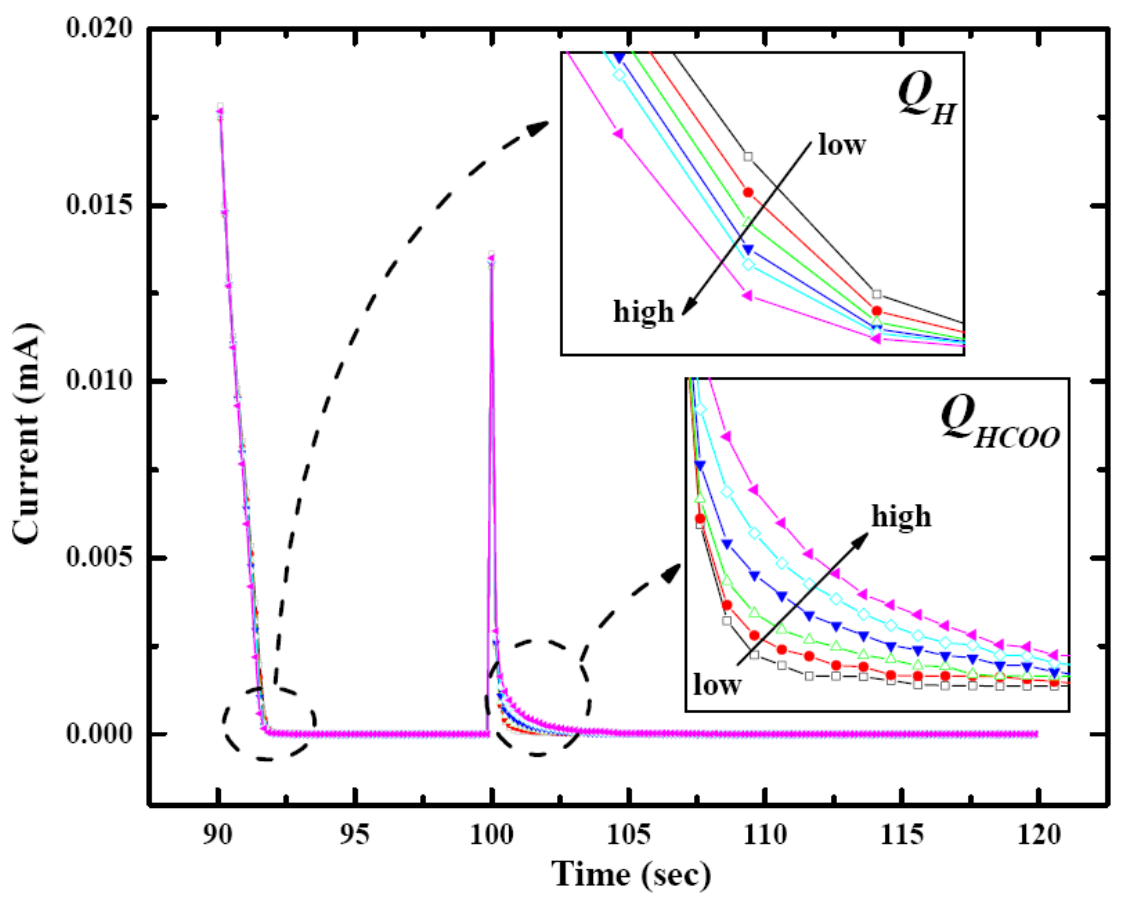


Also depicted in Figure 1 is an i-t curve which illustrates the oxidation charge of the remaining $\mathrm{Pt}-\mathrm{H}\left(Q_{H}\right)$ and the oxidation charge of $\mathrm{Pt}-\mathrm{HCOO}\left(Q_{H C O O}\right)$, defined from each area under the corresponding peak. The first peak starting at $90 \mathrm{~s}$ represented the oxidation of the remaining $\mathrm{Pt}-\mathrm{H}$, while the second peak starting at $100 \mathrm{~s}$ was from the $\mathrm{Pt}-\mathrm{HCOO}$ oxidation. $\mathrm{CO}_{2}$ adsorption occurred only in the presence of $\mathrm{Pt}-\mathrm{H}$, hence the amount of $\mathrm{Pt}-\mathrm{H}$ consumed is correlated to the amount of $\mathrm{CO}_{2}$ that could be adsorbed. A higher amount of $\mathrm{Pt}-\mathrm{HCOO}$ resulted in higher consumption of $\mathrm{Pt}-\mathrm{H}$, therefore the remaining $\mathrm{Pt}-\mathrm{H}$ decreased accordingly as shown in Figure 2. The insets of Figure 2 demonstrate the inversely proportional relationship between two oxidation charges. The schematic of anodic adsorbate stripping of $\mathrm{CO}_{2}$ at low and high concentration illustrating the inverse relationship between $Q_{H}$ and $Q_{H C O O}$ is shown in Figure 3. Typically, only $Q_{H C O O}$ was of interest for the study of $\mathrm{CO}_{2}$ adsorption using the anodic stripping $[19,20]$. However, the inversely proportional relationship between $Q_{H}$ and $Q_{H C O O}$ was found to be essential and informative to explain the dependence of the sensitivity on adsorption potential.

Figure 3. Schematic of anodic stripping of $\mathrm{CO}_{2}$ at low and high concentration illustrating the inverse relationship between $Q_{H}$ and $Q_{H C O O}$.

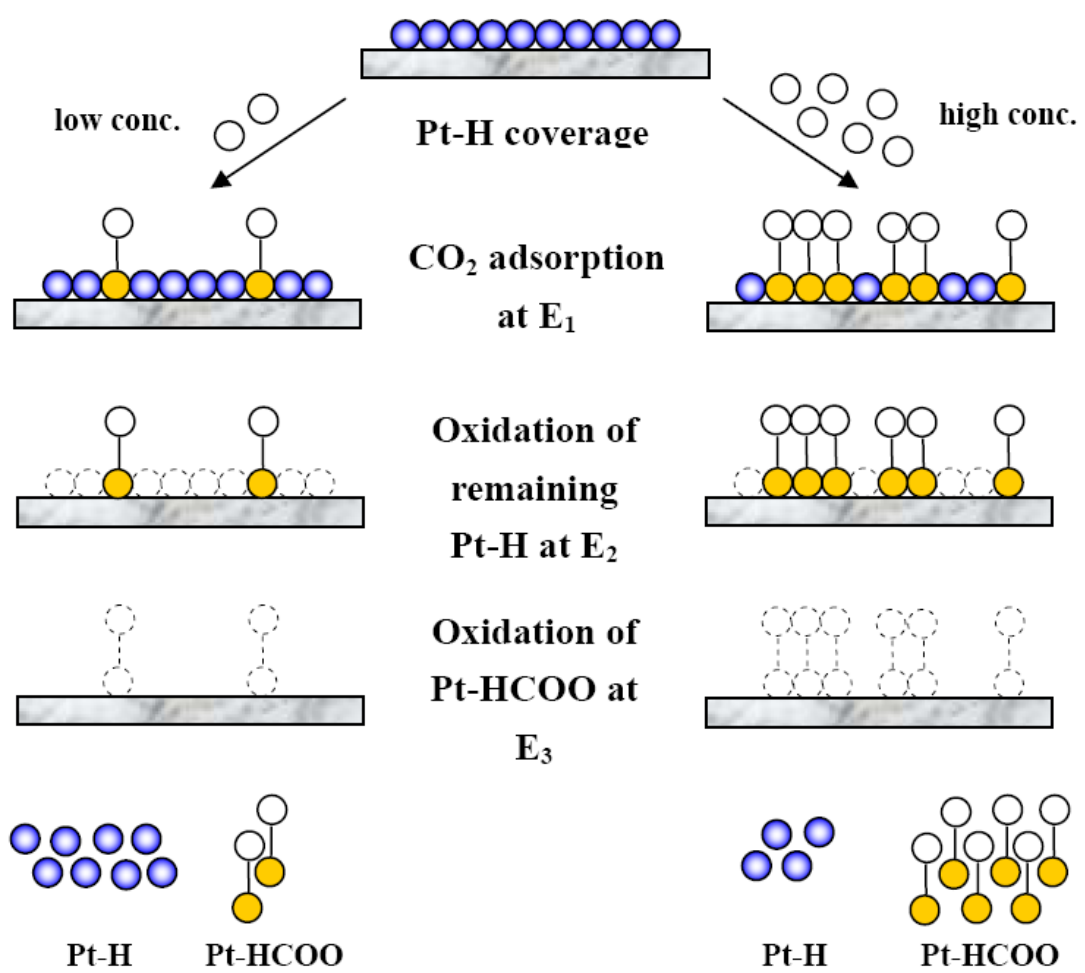

\subsection{Signal Enhancement using Pt Nanoparticles}

The calibration curves for $\mathrm{CO}_{2}$ measurement are shown in Figure 4; the sensing property was found to be enhanced eight-fold employing $\mathrm{Pt}$ nanoparticles. The sensitivity for the $\mathrm{Pt}-\mathrm{Au}$ electrode was $0.030 \mathrm{mC} /\left(\% \mathrm{CO}_{2}\right)$, and for the $\mathrm{Pt}$-nano electrode, $0.239 \mathrm{mC} /\left(\% \mathrm{CO}_{2}\right)$. The linearity of the calibration curves was excellent with $\mathrm{R}^{2}>0.993$. The substantial current increase was due to the increase surface area of the Pt nanoparticles. The ratio of active surface area to geometrical area for 
$\mathrm{Pt}-\mathrm{Au}$ and $\mathrm{Pt}-$ nano electrodes were 1.56 and 20.80, respectively. Consequently, the sensitivity improvement for $\mathrm{CO}_{2}$ detection was anticipated.

Figure 4. Calibration curves for $\mathrm{CO}_{2}$ measurement from chronoamperometry at $+0.75 \mathrm{~V}$ versus $\mathrm{RHE}$, sensitivity of $\mathrm{Pt}-$ nano electrode is eight times higher than that of $\mathrm{Pt}-\mathrm{Au}$ electrode, $\left(-\square_{-}\right) \mathrm{Pt}-\mathrm{Au},(-\bullet-) \mathrm{Pt}-$ nano.

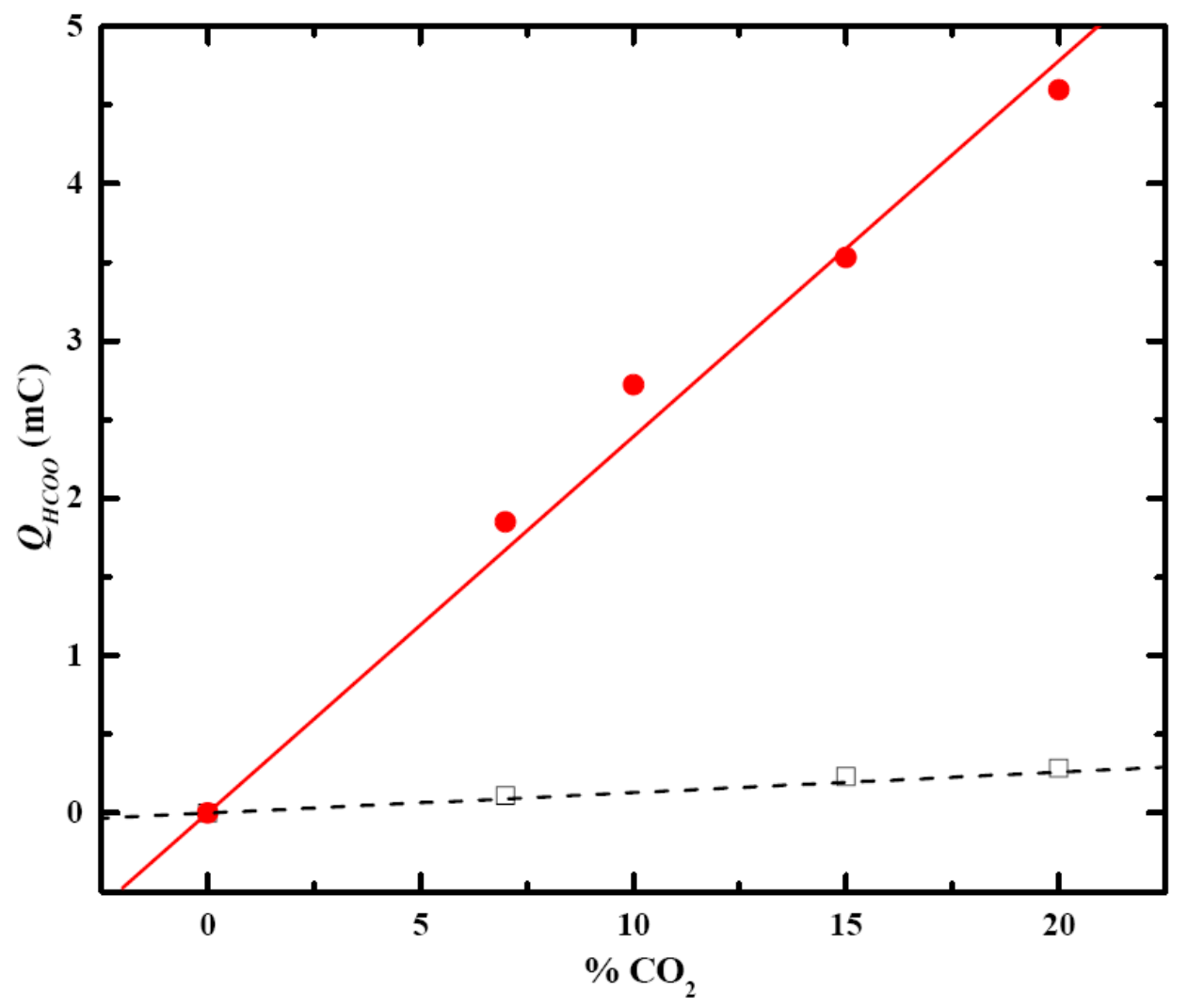

\subsection{Calibration of the Sensor Prototype and Interference Study}

A potential application for the $\mathrm{CO}_{2}$ sensor developed and assessed in this research would be environmental monitoring. Atmospheric air contains $0.038 \% \mathrm{CO}_{2}$, while the concentration of $\mathrm{CO}_{2}$ in exhaled air is approximately 6\%. The OSHA (Occupational Safety and Health Administration) regulations limit $\mathrm{CO}_{2}$ in the workplace to $0.5 \%$ for a prolonged period, and the U.S. National Institute for Occupational Safety and Health limits brief exposures (up to ten minutes) of $\mathrm{CO}_{2}$ to $3 \%$. The detection limit of our sensor prototype is $0.25 \% \mathrm{CO}_{2}$. Thus, the sensing performance of this $\mathrm{CO}_{2}$ sensor was investigated in the lower range (less than $5 \%$ ). Five compositions of $\mathrm{CO}_{2}, 0.5 \%, 1.0 \%, 2.0 \%$, $3.0 \%$ and $5.0 \%$, were prepared using a gas mixture and tested and the corresponding sensor responses are shown in Figure 5. The sensitivity of the sensor for detecting $\mathrm{CO}_{2}$ was $0.249 \mathrm{mC} /\left(\% \mathrm{CO}_{2}\right)$. The calibration curves had an excellent linearity with $\mathrm{R}^{2}>0.994$.

Since the gas sample for the sensor was atmospheric air containing oxygen, nitrogen and other gases in addition to $\mathrm{CO}_{2}$, it is thus necessary to investigate for possible interferences from the other gases. Argon was used to balance the $\mathrm{CO}_{2}$ mixture in the test gas medium instead of air. The sensitivity was found to be $0.240 \mathrm{mC} /\left(\% \mathrm{CO}_{2}\right)$ which was only $3.61 \%$ different from the air-balanced 
gas samples. A good linearity of the calibration curves with $\mathrm{R}^{2}>0.992$ was obtained. Therefore, the sensor response of this developed $\mathrm{CO}_{2}$ sensor demonstrated no significant interference from other gases in ambient air. This is depicted in Figure 5 as well.

Figure 5. Calibration curves Response of $\mathrm{CO}_{2}$ sensor when the sample is balance with, $(-\bullet-)$ air, $(-\bullet)$ argon; there is no significant effect from other gases present in ambient air on $\mathrm{CO}_{2}$ measurement.

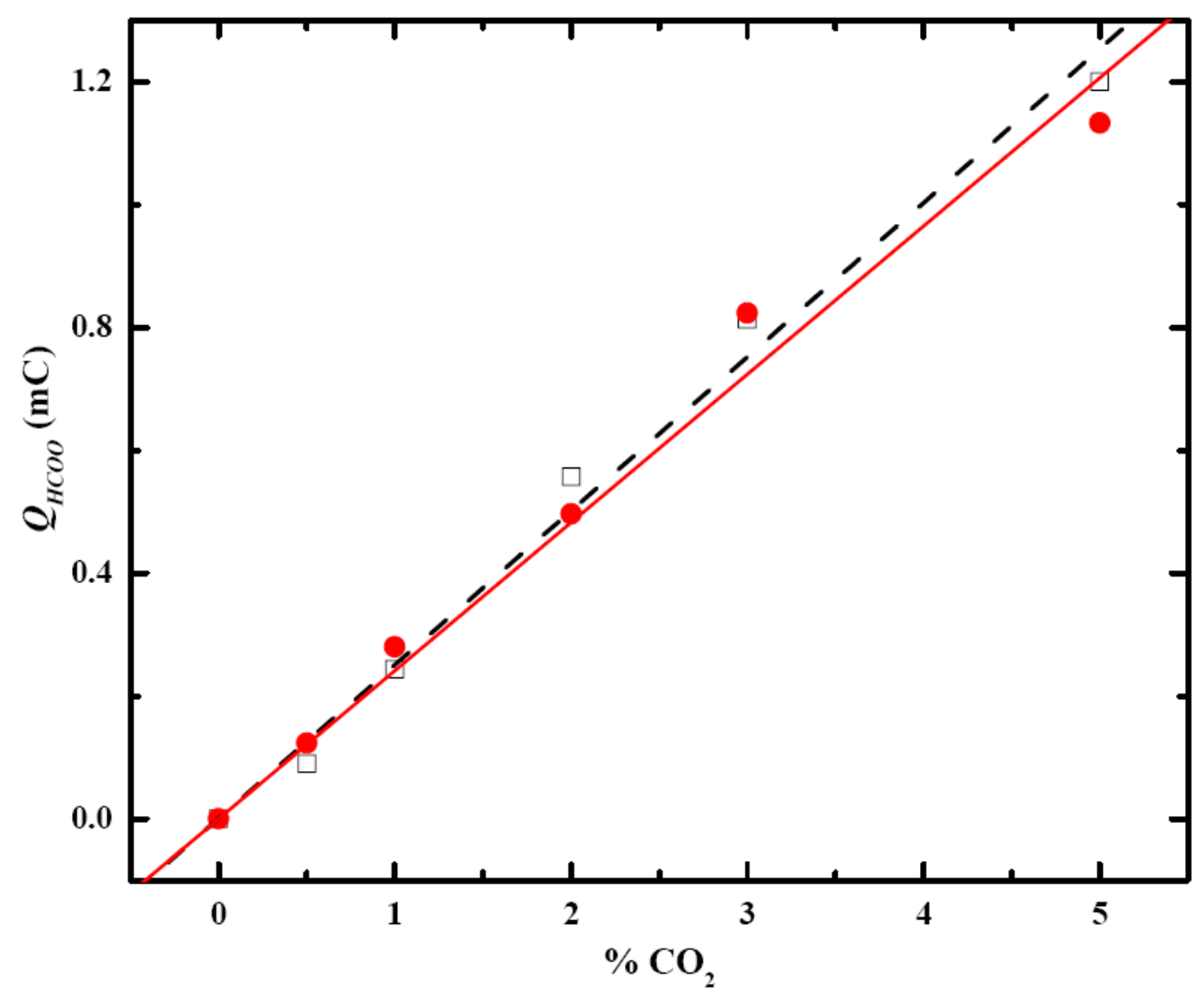

The other possible interferences are carbon monoxide (CO) and organic compounds in the case of harsh environments, which was considered being outside the scope of this work. Nevertheless, since $\mathrm{CO}$ and organics can adsorb on a Pt surface without adsorbed-H and the oxidation of those molecules is irreversible [21], therefore in case of $\mathrm{CO}$ and organics presenting in the system, those molecules could be readily oxidized at potentials above $\mathrm{H}$-adsorption and below surface oxide formation to get rid of any interferences prior to the measurement of $\mathrm{CO}_{2}$.

\subsection{Sensor Stability}

The stability of a $\mathrm{CO}_{2}$ sensor is considered one of its key performance characteristics. In the case of thick-film printing production, good short term stability would be expected rather than in the long term. The active surface area of the sensing electrode elements can be used as an indication of the stability of the sensor. Thus, the measurement of active area of the working electrode was taken after operating the sensor for eight hours. The measurement was carried out on a periodic basis for 14 days. We found that the active electrode surface area continuously declined over the testing period. After 14 days, the 
active area of the working electrode was approximately $8 \%$ less than that of the beginning of the evaluation (result not shown). The stability of this sensor was considered acceptable for thick-film gas sensor, whose other aspects could readily mitigate its moderate stability.

The dependence of the sensitivity on the stability of this $\mathrm{CO}_{2}$ sensor was also studied. A strong dependence was intuitively expected from the fact that sensor response would be proportional to the electrode surface area. The sensor responses for different $\mathrm{CO}_{2}$ content in gas sample on Day 1 and Day 14 were compared. The sensitivities of the sensor for Day 1 and Day 14 were 0.235 and $0.205 \mathrm{mC} /\left(\% \mathrm{CO}_{2}\right)$, respectively. The reduction of sensitivity was less than $13 \%$ (result not shown).

\subsection{Structural Dependence on Sensing Property}

Since the potential for $\mathrm{CO}_{2}$ to adsorb is in the voltage window of hydrogen adsorption potential $(0.00 \mathrm{~V}$ to $0.40 \mathrm{~V}$ versus RHE), an investigation was carried out to obtain the optimum adsorbed potential and the dependence of the quantity of $\mathrm{Pt}-\mathrm{H}$ on the adsorbed potential. The amount of the $\mathrm{Pt}-\mathrm{H}$ produced strongly depends on the adsorption potential as anticipated (result not shown). At a more negative adsorption potential, a higher yield of $\mathrm{Pt}-\mathrm{H}$ was obtained. Experimentally, the range of the adsorption potential between $0.00 \mathrm{~V}$ to $0.15 \mathrm{~V}$ versus RHE was employed. Figure 6(a) shows the behavior of fraction of $\mathrm{Pt}-\mathrm{H}$ being consumed, $\left(1-\theta_{H}\right)$, at different $\mathrm{CO}_{2}$ concentrations.

$$
\begin{gathered}
\theta_{H}=Q_{H} / Q_{H}^{0} \\
1-\theta_{H}=\left(Q_{H}^{0}-Q_{H}\right) / Q_{H}^{0}
\end{gathered}
$$

where $Q_{H}^{0}$ is the oxidation charge of $\mathrm{Pt}-\mathrm{H}$ produced when there is no $\mathrm{CO}_{2}$ present at the corresponding potential. $Q_{H}$ is the oxidation charge of remaining $\mathrm{Pt}-\mathrm{H}$ that are not consumed in $\mathrm{CO}_{2}$ adsorption reaction. Figure 6(b) shows the calibration curve for each adsorption potential.

The dependence of the sensitivity of this $\mathrm{CO}_{2}$ sensor illustrated in Figure 6(b) was not exactly as anticipated. The order of the sensitivity from high to low was $0.10 \mathrm{~V}>0.15 \mathrm{~V}>0.05 \mathrm{~V}>0.00 \mathrm{~V}$ versus RHE. Figure 6(a) showed that the ratio of consumed Pt-H was the highest at $0.10 \mathrm{~V}$, despite having less $\mathrm{Pt}-\mathrm{H}$ produced than at $0.05 \mathrm{~V}$ and $0.00 \mathrm{~V}$. The results from Figure 6(a) suggest that the portion of the $\mathrm{Pt}-\mathrm{H}$ consumed during the $\mathrm{CO}_{2}$ adsorption plays a key role for the sensitivity dependence on the adsorption potential. This particular portion of the $\mathrm{Pt}-\mathrm{H}$ consumed was more reactive than the rest of $\mathrm{Pt}-\mathrm{H}$ on the electrode surface. It was more favorable for $\mathrm{CO}_{2}$ to adsorb on these reactive sites than on the other sites. Evidently, the reactive $\mathrm{Pt}-\mathrm{H}$ was produced most at $0.10 \mathrm{~V}$ because the consumption was the highest. $Q_{H}$ proved to be the pivotal information needed to obtain the observation about the reactive $\mathrm{Pt}-\mathrm{H}$ preferentially produced at $0.10 \mathrm{~V}$. $Q_{H C O O}$ alone was clearly not sufficient to explain the unconventional dependence of the sensitivity on the adsorption potential. The sensor response, hence the sensitivity, at this potential was anticipated to be the highest. A rationale for this observation was made from the dependence of $\mathrm{CO}_{2}$ adsorption on the crystallography of platinum. The polycrystalline Pt nanoparticles consist of three main crystal planes that are of the interest in this study: $\mathrm{Pt}(110), \operatorname{Pt}(100)$, and $\mathrm{Pt}(111)$. The $\mathrm{Pt}(111)$ and $\mathrm{Pt}(100)$ surface has flat structures, while $\mathrm{Pt}(110)$ has step sites that are found to be more reactive to $\mathrm{CO}_{2}$ adsorption than the other two planes [10,22-26]. The structural model also shows that the step sites of $\operatorname{Pt}(110)$ creates a highly 
coordinated configuration that would be more favorable for atoms or molecules to reside, making $\mathrm{Pt}(110)$ more reactive than $\mathrm{Pt}(111)$ and $\mathrm{Pt}(100)$ [27]. Polycrystalline platinum had been studied using $\mathrm{CV}$ [25] and the results showed that the voltammograms exhibited a superimposition of all crystal planes. The $\mathrm{CV}$ voltammogram of a $\mathrm{Pt}$-nano electrode in $0.1 \mathrm{M} \mathrm{H}_{2} \mathrm{SO}_{4}$ is shown in Figure 7(a) and that of a $\mathrm{Pt}-\mathrm{Au}$ electrode in the same medium is shown in Figure 7(b). These results exhibited a similar pattern and it was consistent with other reported results [25]. The peak at $0.10 \mathrm{~V}$ resembles the $\mathrm{Pt}(110)$ pattern which produces the most reactive $\mathrm{Pt}-\mathrm{H}$. Therefore, when the electrode was polarized at $0.10 \mathrm{~V}$, the more reactive $\mathrm{Pt}-\mathrm{H}$ was produced resulting in higher sensor response. This experimental result served well as the rationale for the dependence of sensitivity of the $\mathrm{CO}_{2}$ sensor.

Figure 6. Dependence of (a) $1-\theta_{H}$ and (b) $Q_{H C O O}$ of Pt-nano electrode on adsorption potential, highest $Q_{H C O O}$ and $1-\theta_{H}$ is at $0.10 \mathrm{~V}$ versus RHE ( $\left.\bullet-\right) 0.15 \mathrm{~V},(-\bullet) 0.10 \mathrm{~V}$, $(-\Delta) 0.05,(-\nabla-) 0.00 \mathrm{~V}$.

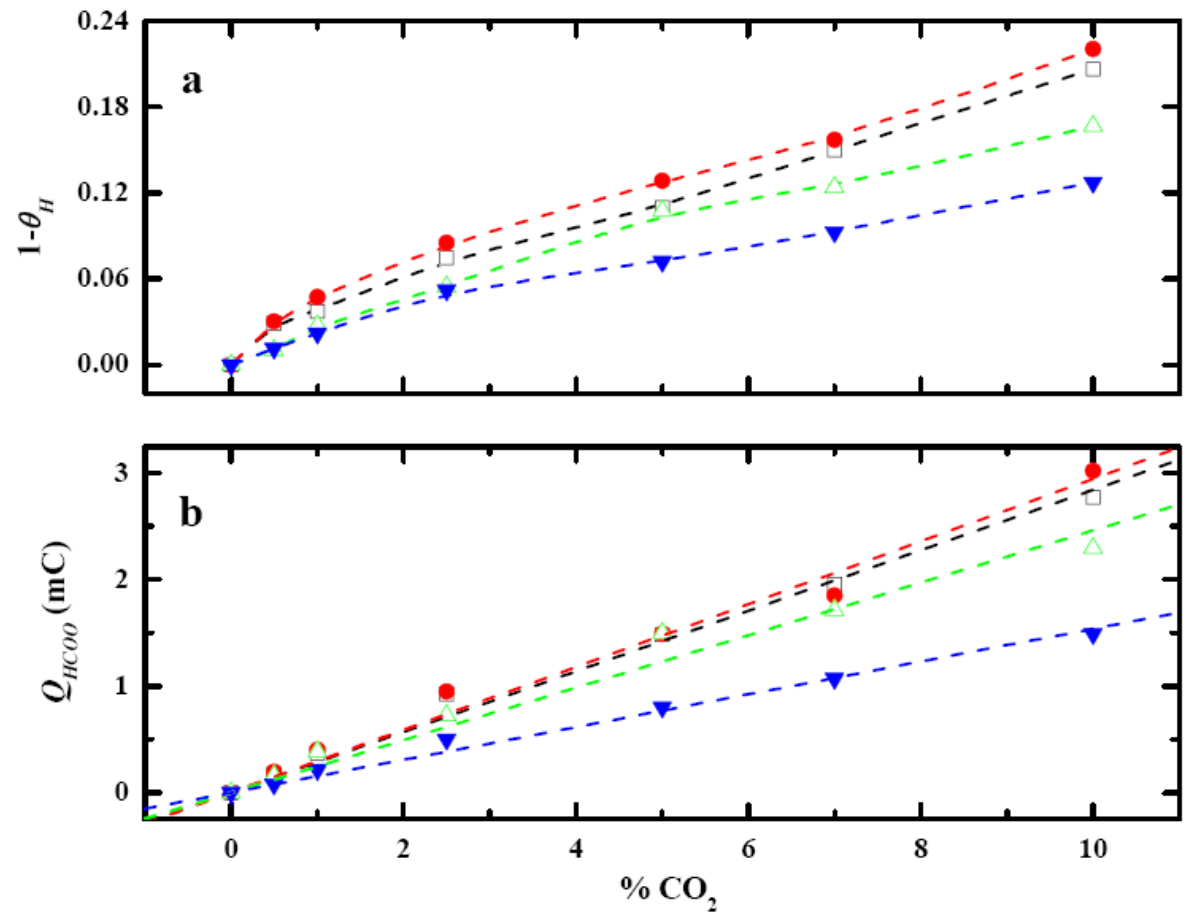

In order to further support this conclusion, studies on the influence of adsorbed potential on $\mathrm{CO}_{2}$ adsorption using a $\mathrm{Pt}-\mathrm{Au}$ electrode were performed. Although the current output of $\mathrm{Pt}-\mathrm{Au}$ was approximately one fifth of the $\mathrm{Pt}-$ nano electrode, the $\mathrm{Pt}-\mathrm{Au}$ electrode also exhibited a similar pattern voltammogram in a $0.10 \mathrm{M} \mathrm{H}_{2} \mathrm{SO}_{4}$ in Figure 7(b). Thus, the behavior of the sensor response and the sensitivity of the $\mathrm{Pt}-\mathrm{Au}$ electrode were expected to be analogous to that of the $\mathrm{Pt}$-nano electrode. As was anticipated, a similar effect of adsorption potential on calibration curves was found and is shown in Figure 8. The sensitivity of the sensor was the highest at the adsorption potential of $0.10 \mathrm{~V}$. The sensitivity of this $\mathrm{Pt}-\mathrm{Au}$ electrode from high to low was in the same order of the $\mathrm{Pt}$-nano electrode, i.e., $0.10 \mathrm{~V}>0.15 \mathrm{~V}>0.05 \mathrm{~V}>0.00 \mathrm{~V}$. The results from the Pt-Au were consistent with the results obtained from the $\mathrm{Pt}-$ nano electrode. Also, reactive $\mathrm{Pt}-\mathrm{H}$ was produced and consumed the most at $0.10 \mathrm{~V}$, which explained the sensitivity dependence on the adsorption potential of the $\mathrm{Pt}-\mathrm{Au}$ electrode. 
Figure 7. Voltammograms of (a) $\mathrm{Pt}$-nano and (b) $\mathrm{Pt}-\mathrm{Au}$ electrodes in $0.10 \mathrm{M} \mathrm{H}_{2} \mathrm{SO}_{4}$ using a scan rate of $50 \mathrm{mV} / \mathrm{s}$, voltammogram resembles the superimposition of three main crystallographic planes. Only peaks from the most two dominant planes revealed; $\operatorname{Pt}(110)$ and $\operatorname{Pt}(100)$.
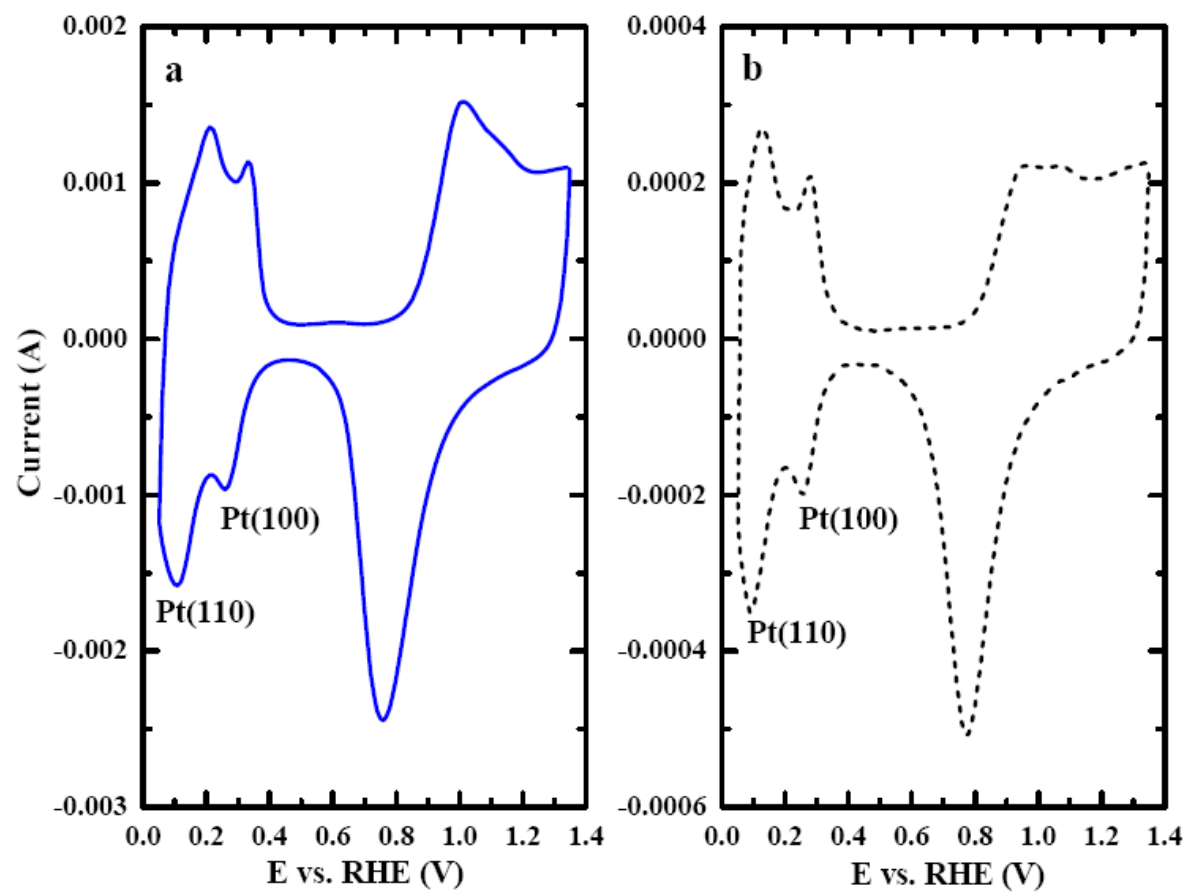

Figure 8. Dependence of (a) $1-\theta_{H}$ and (b) $Q_{\mathrm{HCOO}}$ of $\mathrm{Pt}-\mathrm{Au}$ electrode on adsorption potential, highest $Q_{H C O O}$ and $1-\theta_{H}$ is at $0.10 \mathrm{~V}$ versus RHE ( $\left.\square-\right) 0.15 \mathrm{~V},(-\bullet) 0.10 \mathrm{~V}$, $\left(-\Delta_{-}\right) 0.05,(-\boldsymbol{\nabla}) 0.00 \mathrm{~V}$.
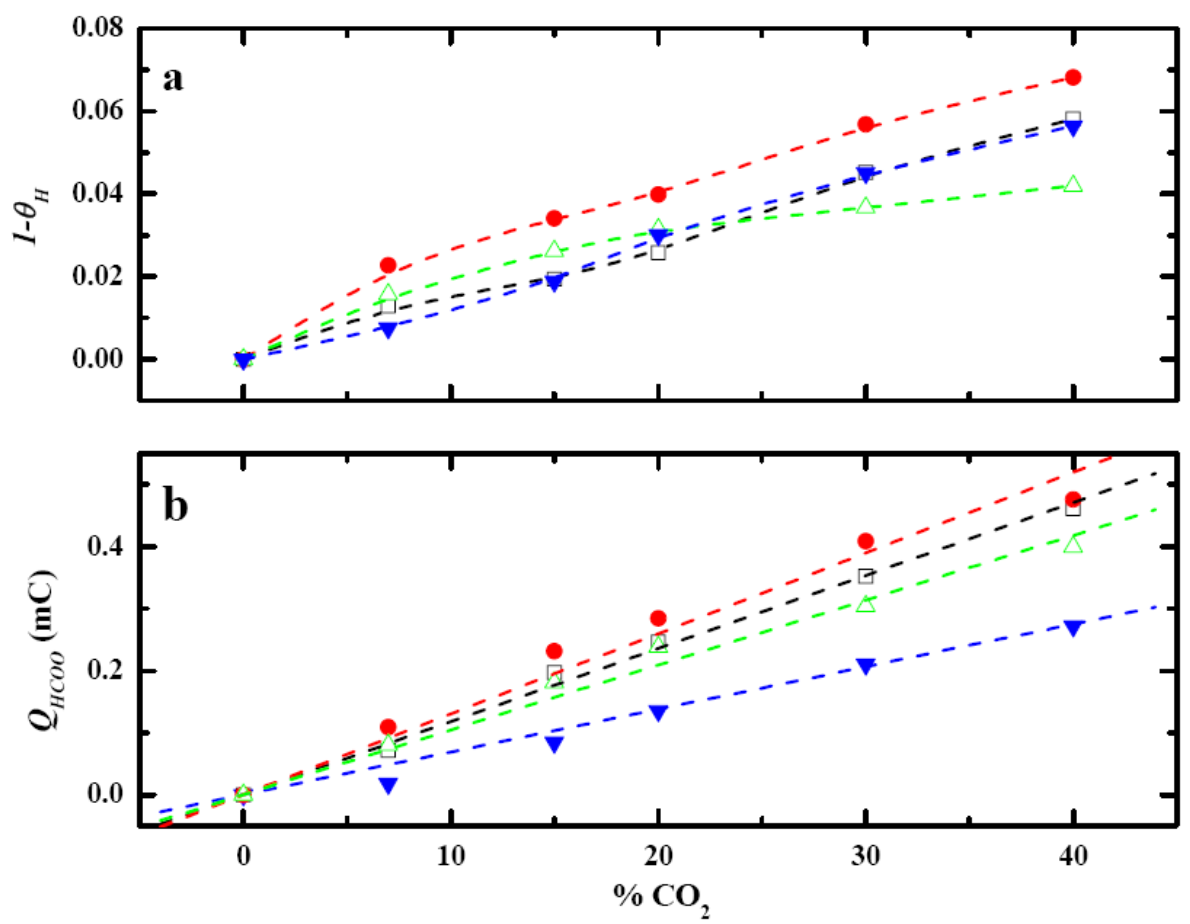


\section{Experimental Section}

\subsection{Sensor Fabrication}

The sensor (Figure 9) was comprised of three electrodes: gold/silver/gold ( $\mathrm{Au} / \mathrm{Ag} / \mathrm{Au}$ ), and was fabricated by thick-film screen printing. The substrate for the sensor prototype was $96 \%$ alumina (Coors, Golden, CO, USA). The metal inks that were used to print the metal leads or electrodes are commercially available. Gold layers were printed first, followed by the silver and the insulation layers. After each layer was printed, the solvent was burnt off at $110{ }^{\circ} \mathrm{C}$ and the substrate was fired at $850{ }^{\circ} \mathrm{C}$ under an ambient air atmosphere. The gold electrode served as the base for the construction of the platinized gold $(\mathrm{Pt}-\mathrm{Au})$ or platinum nanoparticles $(\mathrm{Pt}-\mathrm{nano})$ working electrodes and the platinized gold counter electrode. Pt-Au electrode was formed by electroplating the thick-film gold electrode in a $5 \%$ hexachloroplatinic acid $\left(\mathrm{H}_{2} \mathrm{PtCl}_{6}\right.$, Sigma-Aldrich, St Louis, MO, USA). To platinize gold electrode, an applied potential of $-1.0 \mathrm{~V}$ (versus a Pt gauze) was used and the plating process was approximately 180 seconds. To prepare Pt nanoparticle ink, 3\% w/v hydroxyethyl cellulose (HEC) (Sigma-Aldrich) was first dissolved in $2 \mathrm{~mL}$ DI water, and then $6 \% \mathrm{w} / \mathrm{v}$ polyethylenimine (PEI) (Sigma-Aldrich) was added. Pt nanoparticles in the average diameter of $5.5 \mathrm{~nm}$ (E-TEK) of $10 \% \mathrm{w} / \mathrm{v}$ was added and mixed well using a homogenizer. This Pt nanoparticle ink was the hand-printed on the Pt-Au working electrode surface forming a $2 \mathrm{~mm} \times 7.5 \mathrm{~mm}$ geometric surface area. The sensor was dried for 1 hour and then heated at $125{ }^{\circ} \mathrm{C}$ for 3 hours in an oven under an ambient air atmosphere. The formation of the $\mathrm{AgCl}$ layer on the $\mathrm{Ag}$ base electrode was achieved electrochemically in a $0.10 \mathrm{M} \mathrm{HCl}$ aqueous solution. A potential of $0.50 \mathrm{~V}$ (versus a Pt wire gauze) was applied for 90 seconds. This resulted in a three-electrode based sensor with either a $\mathrm{Pt}-\mathrm{Au}$ or a $\mathrm{Pt}$-nano working electrode, a $\mathrm{Pt}-\mathrm{Au}$ counter electrode and a $\mathrm{Ag} / \mathrm{AgCl}$ reference electrode (Figure 10). All potentials in this paper are referred to RHE.

Figure 9. Three-electrode ( $\mathrm{Au} / \mathrm{Ag} / \mathrm{Au}$ ) sensor layout and its dimensions (in mm).

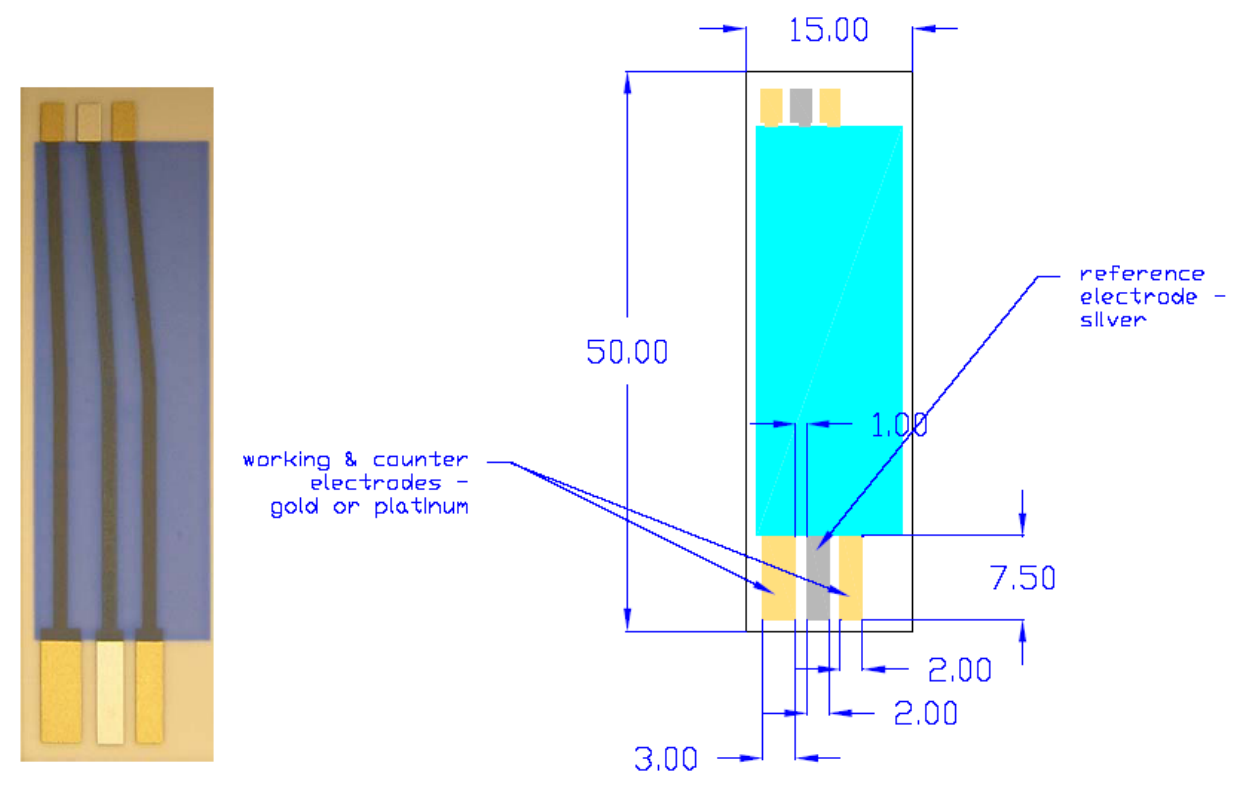


Figure 10. Three-electrode sensor prototypes after platinization, chloridization and hand-printing of Pt nanoparticles ink.

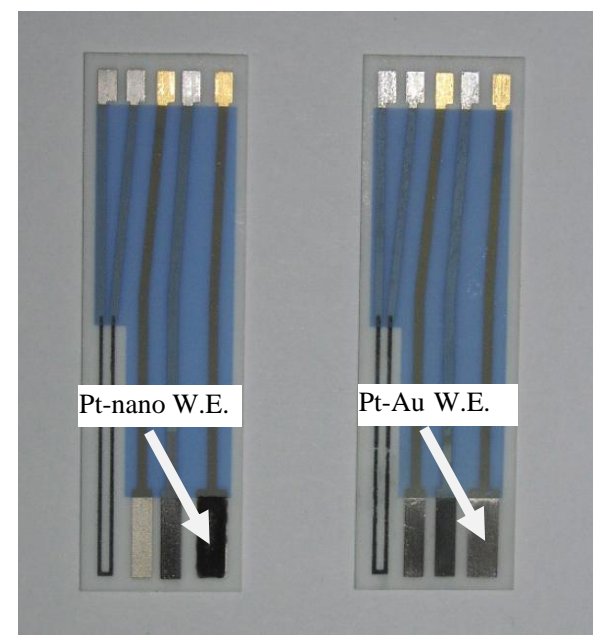

\subsection{Anodic Adsorbate Stripping Technique}

As $\mathrm{Pt}-\mathrm{HCOO}$ has to be formed at a low potential where $\mathrm{Pt}-\mathrm{H}$ is available before undergoing oxidation at higher potential, potential has to be programmed accordingly. From a cyclic voltammogram (Figure 11), the Pt-HCOO is formed in the voltage window of 0.00 to $0.50 \mathrm{~V}$ versus RHE (voltage range of $E_{1}$ and $E_{2}$ ). The oxidation of the Pt-HCOO takes place at $0.75 \mathrm{~V}\left(\mathrm{E}_{3}\right)$. Thus for a $\mathrm{CO}_{2}$ sensor, the platinum working electrode is first held at $0.00 \mathrm{~V}$ for approximately 60 seconds. When a potential of $0.75 \mathrm{~V}$ was applied, the oxidation of $\mathrm{Pt}-\mathrm{HCOO}$ took place and the current produced could then be used to quantify the $\mathrm{CO}_{2}$ presented. The electrolyte for all experiments was $0.10 \mathrm{M} \mathrm{H}_{2} \mathrm{SO}_{4}$.

Figure 11. Cyclic voltammograms of (-) $100 \% \mathrm{CO}_{2}$, and (---) $0 \% \mathrm{CO}_{2}$ in $0.10 \mathrm{M} \mathrm{H}_{2} \mathrm{SO}_{4}$ using a scan rate of $50 \mathrm{mV} / \mathrm{s}$, showing the potential range where $\mathrm{Pt}-\mathrm{HCOO}$ is formed and oxidized.

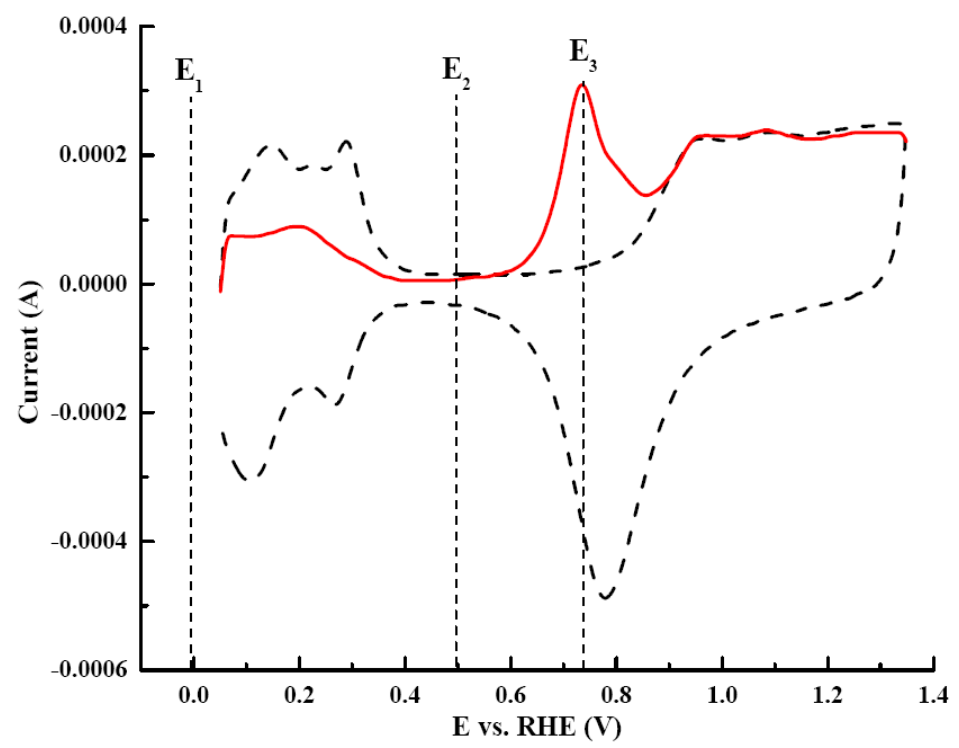




\subsection{Sensing Performance and Interference Study}

To evaluate the sensor performance, the relationship between the sensor outputs and the $\mathrm{CO}_{2}$ concentrations needed to be established. The sensitivity and the reproducibility of this sensor prototype in the $\mathrm{CO}_{2}$ range of $0 \%-20 \%$ were determined. The effect of possible interference by other gases presented in ambient air was also assessed. The main components of ambient air are $\mathrm{N}_{2}$ and $\mathrm{O}_{2}$. Therefore, the results from the samples balanced with air and argon were compared in order to investigate the effect of the interferences.

\subsection{Sensor Stability}

The sensor stability was investigated on a periodic basis for 14 days by comparing the active surface area of the working electrode, which could be used as an indication for the stability of the sensor since the sensor output strongly depends on the surface area of the electrode. The active surface area was calculated from the area under the voltammogram in the hydrogen adsorption region with the appropriate corrections [4,19]. After the sensor was operated for at least eight hours, the voltammogram was recorded and the active surface area was determined. The dependence of the sensor performance on its stability was also studied. The sensitivity of the sensor on Day 1 and Day 14 were compared and the effect of the stability on the sensor was then assessed.

\section{Conclusions}

Our thick-film sensor developed for $\mathrm{CO}_{2}$ detection showed promising performance and stability. The sensor response was enhanced by incorporating Pt nanoparticles onto the working electrode surface. The sensitivity for the Pt-nano electrode was eight times higher than that of the Pt-Au electrode. The sensor also demonstrated good sensitivity and reproducibility. A sensitivity of $0.249 \mathrm{mC} /\left(\% \mathrm{CO}_{2}\right)$ was obtained. Since nitrogen, oxygen and other gases are also present in the gas sample for the potential application, the effects of these gases on the sensor properties were evaluated. The results showed that the response of the sensor was not interfered by these gases. Over 14 days, the active surface area of the working electrode of the sensor decreased approximately $8 \%$ and the sensitivity dropped $13 \%$. Our stability study showed that the sensor would maintain its sensing performance for a reasonably short term use, and the sensor can also be reusable. The thick-film manufacturing technique already offered a costeffective approach to produce these $\mathrm{CO}_{2}$ sensors with acceptable stability and excellent performance.

For structural dependence, it was unexpected that $0.10 \mathrm{~V}$ versus RHE yielded the highest sensitivity instead of $0.00 \mathrm{~V}$, where $\mathrm{Pt}-\mathrm{H}$ produced the most. The crystallographic studies suggested that the quantity of reactive $\mathrm{Pt}-\mathrm{H}$ formed on $\mathrm{Pt}(110)$ was the highest at $0.10 \mathrm{~V}$. This reactive $\mathrm{Pt}-\mathrm{H}$ of $\mathrm{Pt}(110)$ favored $\mathrm{CO}_{2}$ adsorption resulting in the highest sensitivity. A similar assessment was obtained for both $\mathrm{Pt}-$ nano and $\mathrm{Pt}-\mathrm{Au}$ electrodes. Despite of having an order of magnitude difference in the current outputs, $\mathrm{Pt}-$ nano and $\mathrm{Pt}-\mathrm{Au}$ yielded analogous patterns of $\mathrm{CV}$ voltammograms. Therefore, the dependence of the sensitivity of Pt-nano electrode on the adsorption potential was expected to be consistent with that of the $\mathrm{Pt}-\mathrm{Au}$ electrode. The presumed statement was confirmed and illustrated by the results of this study. 


\section{Acknowledgements}

The authors are grateful for financial support from the Royal Thai Government and the Delta Environment and Educational Foundation. We also thank Laurie Dudik for the schematic drawing of a sensor used in Figure 9.

\section{References and Notes}

1. Ishiji, T.; Takahahi, K.; Kira, A. Amperometric carbon dioxide gas sensor based on electrode reduction of platinum oxide. Anal. Chem. 1993, 65, 2736-2739.

2. Evans, J.; Pletcher, D.; Richard, P.; Warburton, G.; Gibbs, T.K. Amperometric sensor of carbon dioxide: Design, charateristics and performance. Anal. Chem. 1989, 61, 577-580.

3. Gilman, S. The mechanism of electrochemical oxidation of carbon monoxide and methanol on platinum. II. The "Reaction Pair" mechanism for electrochemical oxidation of carbon monoxide and methanol. J. Phys. Chem. 1964, 68, 70-80.

4. Gilman, S. The anodic film on platinum electrodes. In Electroanalytical Chemistry, Bard, A.J., Eds.; Marcel Dekker, Inc.: New York, NY, USA, 1967; Vol. 2, pp. 112-189.

5. Giner, J. Electrochemical reduction of $\mathrm{CO}_{2}$ on platinum electrode in acid solutions. Electrochim. Acta 1963, 8, 4164-4167.

6. Sobkowski, J.; Czerwinski, A. The comparative study of $\mathrm{CO}_{2}+\mathrm{H}_{\mathrm{ads}}$ reaction on platinum electrode in $\mathrm{H}_{2} \mathrm{O}$ and $\mathrm{D}_{2} \mathrm{O}$. J. Electroanal. Chem. 1975, 65, 327-333.

7. Sobkowski, J.; Czerwinski, A. Voltammetric study of $\mathrm{CO}$ and $\mathrm{CO}_{2}$ adsorption on smooth and platinized platinum electrodes. J. Phys. Chem. 1985, 89, 365-369.

8. Baruzzi, A.M.; Leiva, E.P.M.; Giordano, M.C. Complex kinetic behaviour of "reduced" $\mathrm{CO}_{2}$ electro-oxidation at Pt electrodes. J. Electroanal. Chem. 1983, 158, 103-114.

9. Brummer, S.B.; Cahill, K. Kinetics of reductive adsorption of $\mathrm{CO}_{2}$ on smooth Pt electrodes. $J$. Electroanal. Chem. 1969, 21, 463-469.

10. Hoshi, N.; Mizumura, T.; Hori, Y. Significant difference of the reduction rates of carbon dioxide between Pt(111) and Pt(110) single crystal electrodes. Electrochim. Acta 1995, 40, 883-887.

11. Hoshi, N.; Noma, M.; Suzuki, T.; Hori, Y. Structural effect on the rate of $\mathrm{CO}_{2}$ reduction on single crystal electrodes of palladium. J. Electroanal. Chem. 1997, 421, 15-18.

12. Willsau, J.; Heitbaum, J. Analysis of adsorbed intemediates and determination of surface potential shifts by DEMS. Electrochim. Acta 1986, 31, 943-948.

13. Sobkowski, J.; Czerwinski, A. Kinetics of carbon dioxide adsorption on a platinum electrode. $J$. Electroanal. Chem. 1974, 59, 41-46.

14. Brisard, G.M.; Camargo, A.P.M.; Nart, F.C.; Iwasita, T. On-line mass spectrometry investigation of the reduction of carbon dioxide in acidic media on polycrystalline Pt. Electrochem. Commun. 2001, 3, 603-607.

15. Hoshi, N.; Suzuki, T.; Hori, Y. $\mathrm{CO}_{2}$ reduction on $\mathrm{Pt}(\mathrm{S})-(\mathrm{n}(111) \mathrm{X}$ (111)I single crystal electrodes affected by the adsorption of sulfuric acid anion. J. Electroanal. Chem. 1996, 416, 61-65. 
16. Sremaniak, L.S.; Whitten, J.L. Theoretical treatment of excited electronic states of adsorbates on metals: Electron attachment to CO2 adsorbed on K-modified Pt(1 11 1). Surface Sci. 2008, 602, 834-842.

17. de Bruijn, F.A.; Papageorgopoulos, D.C.; Sitters, E.F.; Janssen, G.J.M. The influence of carbon dioxide on PEM fuel cell anodes. J. Power Sources 2002, 110, 117-124.

18. Sremaniak, L.S.; Whitten, J.L. Theoretical treatment of excited electronic states of adsorbates on metals: Electron attachment to CO2 adsorbed on Pt(1 1 1). Surface Sci. 2007, 601, 3755-3759.

19. Kuver, A.; Vielstich, W.; Kitzelmann, D. On the quantitative determination of carbon dioxide in air. A new sensor technique using anodic adsorbate stripping. J. Electroanal. Chem. 1993, 353, 255-263.

20. Wang, Y.; Yan, H.; Liu, J. Solid polymer electrolyte-based $\mathrm{CO}_{2}$ sensor using anodic adsorbate stripping. Anal. Lett. 2005, 38, 2057-2065.

21. Siwek, H.; Tokarz, W.; Piela, P.; Czerwinski, A. Electrochemical Behavior of CO, CO2 and Methanol Adsorption Products Formed on Pt-Rh Alloys of Various Surface Composition. J. Power Sources 2008, 181, 24-30.

22. Feddrix, F.H.; Yeager, E.B.; Cahan, B.D. Low energy electron diffraction and cyclic voltammetry studies of flame-annealed platinum single crystals. J. Electroanal. Chem. 1992, 330, 419-431.

23. Hoshi, N.; Hori, Y. Electrochemical reduction of carbon dioxide at a series of platinum single crystal electrodes. Electrochim. Acta 2000, 45, 4263-4270.

24. Hoshi, N.; Suzuki, T.; Hori, Y. $\mathrm{CO}_{2}$ reduction on $\mathrm{Pt}(\mathrm{S})-(\mathrm{n}(111) \mathrm{X}$ (111)I single crystal electrodes affected by the adsorption of sulfuric acid anion. J. Electroanal. Chem. 1996, 416, 61-65.

25. Mendez, E.; Martins, M.E.; Zinola, C.F. New effects in the electrochemistry of carbon dioxide on platinum by the application of potential perturbations. J. Electroanal. Chem. 1999, 477, 41-51.

26. Rodes, A.; Pastor, E.; Iwasita, T. Structural effects on $\mathrm{CO}_{2}$ reduction at $\mathrm{Pt}$ single-crystal electrodes. Part 1. The Pt(110) surface. J. Electroanal. Chem. 1994, 369, 183-191.

27. Yamamoto, K.; Kolb, D.M.; Kotz, R.; Lehmpfuhl, G. Hydrogen absorption and oxide formation on platinum single crystal electrodes. J. Electroanal. Chem. 1979, 96, 233-239.

(C) 2009 by the authors; licensee Molecular Diversity Preservation International, Basel, Switzerland. This article is an open-access article distributed under the terms and conditions of the Creative Commons Attribution license (http://creativecommons.org/licenses/by/3.0/). 